\title{
Trial for Preparation and Evaluation of Combined Inactivated Vaccine for the Protection against Riemerella anatipestifer and Avian Influenza (H5N1) in Ducks
}

\author{
Fatma El-Zahraa Gamal ${ }^{1}$, Eman M. Soliman**, Heba M. El-Naggar², Wafaa S. Abd El- \\ MONEIM $^{2}$, AmR IsMail Hassannin ${ }^{2}$
}

${ }^{1}$ Central Laboratory for Evaluation of Veterinary Biologics, Abbasia, Agricultural Research Center (CLEVB/ARC), Cairo, Egypt; ${ }^{2}$ Veterinary Serum and Vaccine Research Institute, Abbasia, Agricultural Research Center (VSVRI) $A R C$ ), Cairo, Egypt.

\begin{abstract}
Riemerella anatipestifer and Avian Influenza infections cause major economic losses in the duck industry through high mortality rates, poor feed conversion, increased condemnations, and high treatment costs. A field trial was conducted to evaluate the immunogenicity of an experimentally multivalent inactivated $R$. anatipestifer and Avian Influenza (H5N1) vaccine. In this study a combined inactivated Montanide ISA $71 R$. anatipestifer and Avian Influenza (H5N1) vaccine was prepared then the immunization potency and protective efficacy were evaluated through experimental application on Pekin ducklings. The peak of humeral immune response against $R$. anatipestifer as measured by indirect haemagglutination inhibition test $(\mathrm{HI})$ was at the $6^{\text {th }}$ week post primary vaccination while it was at the $4^{\text {th }}$ week when boostering 3 weeks after primary vaccination and $3^{\text {rd }}$ week when boostering 8 weeks after primary vaccination; meanwhile $\mathrm{AI}$ immune response as measured by $(\mathrm{HI})$ reached the peak at the $5^{\text {th }}$ week post primary vaccination and at $4^{\text {th }}$ week for single $\mathrm{AI}$ and $5^{\text {th }}$ week for combined RA and $\mathrm{AI}$ vaccines when boostering 3 weeks after primary vaccination and $5^{\text {th }}$ week when boostering 8 weeks after primary vaccination . Regarding the challenge test, the protection percentage were $70 \%$ against both $\mathrm{RA}_{1}$ and $\mathrm{RA}_{2}$ virulent strains in $\mathrm{RA}$ vaccine and $75 \%$ in combined $\mathrm{RA}$ and $\mathrm{AI}$ vaccine after challenge 3 weeks post primary vaccination, while it was $80 \%$ against $R A_{1}$ and $R A_{2}$ virulent strains in RA vaccine and $85 \%$ in combined RA and $\mathrm{AI}$ vaccine after challenge 3 weeks post boostering. In conclusion the combined prepared $R$. anatipestifer and Avian Influenza (H5N1) vaccine succeeded in eliciting protective antibody titers and satisfactory protection against both $R$. anatipestifer and Avian Influenza (H5N1) diseases.
\end{abstract}

Keywords | R. anatipestifer, AI, vaccine; HI, Challenge test

Received | December 23, 2020; Accepted | January 01, 2021; Published | February 15, 2021

*Correspondence | Eman M Soliman, Central Laboratory for Evaluation of Veterinary Biologics, Abbasia, Agricultural Research Center (CLEVB/ARC), Cairo, Egypt; Email: emansoliman1880@gmail.com

Citation | Gamal FZ, Soliman EM, El-Naggar HM, Abd El-Moneim WS, Hassannin AI (2021). Trial for preparation and evaluation of combined inactivated vaccine for the protection against riemerella anatipestifer and avian influenza (h5n1) in ducks. Adv. Anim. Vet. Sci. 9(4): 490-499.

DOI | http://dx.doi.org/10.17582/journal.aavs/2021/9.4.490.499

ISSN (Online) | 2307-8316; ISSN (Print) | 2309-3331

Copyright (c) 2021 Soliman et al. This is an open access article distributed under the Creative Commons Attribution License, which permits unrestricted use, distribution, and reproduction in any medium, provided the original work is properly cited.

\section{INTRODUCTION}

$R$ iemerella anatipestifer and Avian Influenza cause major economic losses in duck industry through high mortality rates, poor feed conversion, increased condemnations, and high treatment costs (Kardos et al., 2007; Sun et al., 2012). $R$. anatipestifer infection causes mortality rates between 10 and $75 \%$ in ducks fewer than 8 weeks of age in the infected farms (Subramaniam et al., 2000). Now a day,
21 serotypes have been identified in the world, and there is poor cross-protection among these serotypes (Kardos et al., 2007). Infections from $R$. anatipestifer serotypes 1, 2, 3, $5,6,7,8,10,11,13,14$, and 15 have been reported in China; however, serotypes 1,2 are responsible for most of the major outbreaks (Hu et al., 2001). Signs usually include head shaking, lethargy and an abnormal gait. Post mortem signs in acute cases include enlargement of the liver and spleen and lung congestion. In more chronic cases, pericar- 
ditis, perihepatitis and air sacculitis with a caseous deposit can be found (Sandhu, 1986; Higgins et al., 2000).

Various antibiotics are currently used to prevent and control $R$. anatipestifer infection in ducks, but they accelerate the emergence of drug-resistant strains (Chen et al., 2010; Chen et al., 2012). The resistance of pasteurella multoci$d a$ and $R$. anatipestifer for many antibiotics has increased greatly, and antibiotic residues have been detected in duckrelated products (Sun et al., 2012). So, vaccine application is the most accepted method for the control of the diseases, by obtaining a high level of antibodies, rather than using other eradication or medication strategies. Vaccines based on a single serotype of inactivated bacterin have not provided significant cross- protection among the serotypes (Sandhu, 1979).

Highly pathogenic avian influenza virus (HPAIV) H5N1 $(\mathrm{A} / \mathrm{H} 5 \mathrm{~N} 1)$ caused enormous economic losses in poultry in many countries worldwide and genetically diversified into 10 clades and several subclades since 1996/1997 (Smith and Donis, 2015). EgyptianA/H5N1 of clade 2.2.1 has diversified into several genetic groups. Most of these phylogroups disappeared but two major clades circulated for several years (Naguib et al., 2016a). Clade 2.2.1.1 represented antigenic-drift variants, which were primarily isolated from vaccinated commercial poultry leading to three human infections so far according to the official reports to the (WHO, 2017). These viruses appeared in early 2007 and predominated in 2008-2010 challenging the efficacy of the highly diverseH5 vaccines in Egypt. In 2011-2014, the prevalence of 2.2.1.1 viruses dramatically decreased and they are most likely extinct by now (Naguib et al., 2016b; E1-Shesheny et al., 2017; Rohaim et al., 2017). The second clade is 2.2.1.2 viruses which circulated in non-vaccinated backyard birds and were introduced into small-scale farmed poultry since early 2008 . The vast majority of infected humans were infected by this genotype (Younan et al., 2013). In 2014/2015, an unprecedented upsurge of 2.2.1.2 was reported in poultry and humans marking Egypt as the country with the highest number of human infections with A/ H5N1 worldwide (Arafa et al., 2015; WHO, 2017). These viruses spread to neighboring countries posing a serious threat in the Middle East (Naguib et al., 2016a; Salaheldin et al., 2017). Driving forces for the emergence, extinction or spread of A/H5N1 clades in Egypt are not well-studied except for the massive application of vaccines and antivirals in poultry (Abdel- Moneim et al., 2011; Cattoli et al., 2011).Improved vaccination strategy is required to increase the immunogenicity in chicken farms. These strategies could include: the use of a highly immunogenic vaccine seed strain, the improvement of an effective adjuvant for chickens, and the consideration of a 2-dose regimen (Dong-Hun Lee et al., 2013). To reach optimal protec- tion, the vaccine should be matching to the field strain and reduced shedding of the virus. As the antigenic drift of AI viruses after extended vaccination programs has been observed in chickens, similar to what has been observed with human influenza viruses (Suarez et al., 2006). Using a recently developed plasmid-based reverse genetics system for influenza, viruses can be rescued from cloned cDNA that enables the rapid generation of reassortant influenza virus, using a plasmid-only rescue system, (Hoffmann et al., 2002) described the usefulness of the reverse genetics system for the rapid generation of reassortants for human influenza vaccine.. In addition, it can convert High pathogenic avian influenza (HPAI) into Low pathogenic avian Influenza (LPAI) vaccine candidates by mutating the HA cleavage site, allowing an easier and safer handling for vaccine manufactures (Subbarao and Kafz, 2004). Two AI-inactivated vaccines (a homologous $\mathrm{H} 5 \mathrm{~N} 1$ and a heterologous H5N3) using this technology have recently been licensed (Liu et al., 2003). These vaccines which contain a homologous HA gene have been shown to be more protective in ducks and geese than conventional vaccines with a more distant HA gene (Middleton et al., 2007).

Because of both Avian influenza and $R$. anatipestifer infections cause economic losses, occur at about the same age, and have same signs and post-mortem lesions, the aim of work is the development of a combined inactivated vaccine against $R$. anatipestifer and $\mathrm{AI}$ as a convenient product for breeding stock, where it can be injected in one shot for the purposes of covering the above mentioned points, it is suggested that such vaccine could be able to protect ducks against field challenges exhibiting them good immunity levels which can be passed to the next generation through the egg yolk.

\section{MATERIALS AND METHODS}

\section{STRAINS USED IN VACCINE PREPARATION}

Riemerella anatipestifer: Riemerella anatipestiferthe local isolates of $\mathrm{RA}_{1}$ and $\mathrm{RA}_{2}$ were obtained from Aerobic bacterial vaccines department in VSVRI/ARC Egypt and previously identified morphologically, biochemically and genetically.

Avian Influenza $\mathbf{H}_{5} \mathbf{N}_{1}$ : For $\mathrm{H} 5 \mathrm{~N} 1$ vaccine strain preparation (RGD10552B/H5N1), a plasmid-based reverse genetics system was applied using 6 internal genes of $\mathrm{A} /$ Puerto Rico/8/34(H1N1) and 2 surfaces LP-HA and NA genes of D10552B/H5N1 virus. Field isolate attenuated by reverse genetics and provided from National Research Center/ Egypt. (Mokhtar et al., 2019).

\section{Adjuvant}

Montanide $^{\mathrm{TM}}$ ISA 71 VG. It is a mineral oil based adju- 
vant that has been developed for manufacture of water-inoil (W/O) emulsion. The adjuvant was kindly provided by SEPPIC Co, France.

\section{VACCINE PREPARATION}

Preparation of inactivated Riemerella anatipestifer antigenic phase: It was according to (Pathanasophon et al., 1996): $R$. anatipestifer isolates were cultured separately in Tryptic Soya Broth (TSB) at $37^{\circ} \mathrm{C}$ for 24 hours with shaking. The count of bacterial CFU for each strain was adjusted to $1.4 \times 10^{10} \mathrm{CFU} / \mathrm{ml}$ for each one. The bacteria were then inactivated with $0.5 \%$ formalin at $37^{\circ} \mathrm{C}$ for 24 hours. After inactivation each culture was tested for purity, safety and sterility as mentioned by (OIE, 2019). Finally, cultures were equally mixed together then preserved with $0.01 \%$ of thiomersal and stored at $4^{\circ} \mathrm{C}$ until used.

Preparation of inactivated $\mathrm{AIH}_{5} \mathrm{~N}_{1}$ antigenic phase: Propagation and titration of $\mathrm{AI} \mathrm{H}_{5} \mathrm{~N}_{1}$ were done in $\mathrm{SPF}$ eggs 9 -10 day old Embryonated Chicken Egg (ECE) according to (OIE, 2019). Its titer was $10^{9} \mathrm{EID}_{50} / \mathrm{ml}$. Inactivation of $\mathrm{AI}$ virus was carried out using formalin with final concentration $0.01 \%$ of total volume at $25^{\circ} \mathrm{C}$ for 18 hrs. After inactivation sodium bisulfite was added as a final concentration of $2 \%$ to stop the action of formalin and stored at $4^{\circ} \mathrm{C}$ until used. The antigen content in all the prepared vaccines must be not less than $10^{8.5}$ embryo infective dose $50 \%\left(\mathrm{EID}_{50}\right)$. Inactivation and testing of inactivation was done according to (OIE, 2019).

Formulation of inactivated Riemerella anatipestifer, inactivated Avian influenza and combined inactivated Riemerella anatipestifer and AI vaccines: The inactivated Riemerella anatipestifer, the inactivated avian influenza and the combined inactivated Riemerella anatipestifer and AI vaccines were prepared by the formulation process recommended by Montanide manufacture. W/O emulsion vaccine using Montanide ${ }^{\mathrm{TM}}$ ISA $71 \mathrm{VG}$ at a ratio of $30 / 70$ (W/W) aqueous/oil ratio was prepared. The same for combined inactivated $R$. anatipestifer and AI vaccines but we added equal weight for both $\mathrm{AI} \& R$. anatipestifer taking in consideration that the antigenic content for both antigens not less than $10^{8} \mathrm{EID}_{50}$. Stable preparations were obtained by mixing the aqueous medium into the Montanide ${ }^{\mathrm{TM}}$ ISA $71 \mathrm{VG}$, at room temperature or less, under vigorous stirring (for 15-30 $\mathrm{min}$ ) as recommended by manufacture.

\section{QUALITY CONTROL OF THE PREPARED VACCINE}

The prepared vaccines were tested for sterility, safety and purity tests following the standard international protocols as described by (CFR, 2019).

\section{EXPERIMENTAL DUCKS}

Two hundred and fifty, one day-old apparently healthy Pe- kin ducklings obtained from commercial farm at El-Minufiya Governorate were used in this study. The ducks were kept and reared in separate pens at the animal husbandry facilities of Central Laboratory for Evaluation Veterinary Biologics (CLEVB) until used for the experiment. Blood samples were collected directly before the experiment and serological examinations (IHA, HI) were done to ensure that the ducks have no maternal or acquired antibodies against $R$. anatipestifer or AI.

\section{EXPERIMENTAL DESIGN}

A total of 250 ducklings four weeks old were divided into four groups, the 1 st group (90 ducks) was vaccinated with single dose of the prepared inactivated oil emulsion $R$. anatipestifer vaccine. The 2 nd group ( 20 ducks) was vaccinated with single dose of the prepared inactivated oil emulsion AI vaccine. The 3rd group (90 ducks) was vaccinated with single dose of the prepared inactivated oil emulsion combined $R$. anatipestifer and AI vaccine. The 4th group (50 ducks) was remained as control unvaccinated group.

The 1st and 3rd groups were divided into 3 subgroups and inoculated as follow:

Subgroup (a) (40 ducks): received single dose then challenged 3weeks post vaccination. Subgroup (b) (40 ducks): received first dose and booster dose 3 weeks post vaccination then challenged 3 weeks post boostering. Subgroup (c) (10 ducks): received single dose and the titer was followed up until decreased then received booster dose.

The $2^{\text {nd }}$ group was divided into two equal subgroups, subgroup (a) (10 ducks): received first dose and booster dose 3 weeks post vaccination and subgroup (b) (10 ducks) received single dose and the titer was followed up until decreased then received booster dose. Table (1).

\section{VACCINE INOCULATION}

The ducks in each group were inoculated $\mathrm{S} / \mathrm{C}$ with $0.5 \mathrm{ml}$ of vaccines at the dorsal aspect of the neck.

\section{BLOOD SAMPLES COLLECTION}

Random blood samples were collected weekly post immunization to evaluate the developed immune response to the vaccines.

SEROlogical tests FOR EVALUATION OF HUMERAL IMMUNE RESPONSE OF PREPARED VACCINES

Indirect haemagglutination test (IHA): IHA test was used for evaluation of humeral immune response against $R$. anatipestifer. The glutraldehyde RBCs and capsular antigen of $R$. anatipestifer were prepared according to (Carter and Cole, 1990). A vaccine is considered efficient as induce sera conversion in the sera of vaccinated ducks. 
Table 1: The experimental design

\begin{tabular}{|c|c|c|c|c|c|c|c|c|}
\hline Groups & \multicolumn{3}{|l|}{1} & \multicolumn{2}{|l|}{2} & \multicolumn{3}{|l|}{3} \\
\hline Vaccines & \multicolumn{3}{|c|}{ Inactivated $R A$ vaccine } & \multicolumn{2}{|c|}{ Inactivated AI vaccine } & \multicolumn{3}{|c|}{$\begin{array}{l}\text { Combined inactivated } R A \text { and } A I \\
\text { vaccine }\end{array}$} \\
\hline Subgroups & c & $\mathrm{b}$ & $\mathrm{a}$ & $\mathrm{b}$ & $\mathrm{a}$ & c & $\mathrm{b}$ & $\mathrm{a}$ \\
\hline & $\begin{array}{l}\text { Received } \\
\text { single dose } \\
\text { and the } \\
\text { antibody titer } \\
\text { was followed } \\
\text { till decreased } \\
\text { then received } \\
\text { booster dose }\end{array}$ & $\begin{array}{l}\text { Received } \\
\text { first dose } \\
\text { then booster } \\
\text { dose } 3 \\
\text { weeks after } \\
\text { vaccina- } \\
\text { tion then } \\
\text { challenged } 3 \\
\text { weeks after } \\
\text { boostering }\end{array}$ & $\begin{array}{l}\text { Received } \\
\text { single } \\
\text { dose then } \\
\text { challenged } \\
3 \text { weeks } \\
\text { after vac- } \\
\text { cination }\end{array}$ & $\begin{array}{l}\text { Received } \\
\text { single dose } \\
\text { and the } \\
\text { antibody } \\
\text { titer was } \\
\text { followed till } \\
\text { decreased } \\
\text { then } \\
\text { received } \\
\text { booster } \\
\text { dose }\end{array}$ & $\begin{array}{l}\text { Received } \\
\text { first dose } \\
\text { then booster } \\
\text { dose } 3 \\
\text { weeks after } \\
\text { vaccina- } \\
\text { tion then } \\
\text { challenged } 3 \\
\text { weeks after } \\
\text { boostering }\end{array}$ & $\begin{array}{l}\text { Received } \\
\text { single dose } \\
\text { and the } \\
\text { antibody titer } \\
\text { was followed } \\
\text { till decreased } \\
\text { then received } \\
\text { booster dose }\end{array}$ & $\begin{array}{l}\text { Received } \\
\text { first dose } \\
\text { then booster } \\
\text { dose } 3 \\
\text { weeks after } \\
\text { vaccina- } \\
\text { tion then } \\
\text { challenged } 3 \\
\text { weeks after } \\
\text { boostering }\end{array}$ & $\begin{array}{l}\text { Received } \\
\text { single } \\
\text { dose then } \\
\text { challenged } \\
3 \text { weeks } \\
\text { after vac- } \\
\text { cination }\end{array}$ \\
\hline
\end{tabular}

RA: Riemerella anatipestifer

AI: Avian influenza

Table 2: IHA antibody titers in sera of ducklings vaccinated with single dose of inactivated $R$. anatipestifer and combined $R$. anatipestifer and $\mathrm{AI}$ vaccines

\begin{tabular}{llllll} 
Weeks post primary vaccination & \multicolumn{2}{l}{$\begin{array}{l}\text { Type of vaccines } \\
\text { Inactivated RA vaccine }\end{array}$} & \multicolumn{2}{l}{ Combined inactivated RA and AI vaccine } & Control \\
& $\mathrm{RA}_{1}$ & $\mathrm{RA}_{2}$ & $\mathrm{RA}_{1}$ & $\mathrm{RA}_{2}$ & \\
\hline Pre-vaccination & 2 & 2 & 2 & 2 & 0.0 \\
$2^{\text {nd }}$ week & 16 & 8 & 16 & 16 & 0.0 \\
\hline $3^{\text {rd }}$ week & 16 & 16 & 32 & 32 & 0.0 \\
$4^{\text {th }}$ week & 32 & 32 & 64 & 64 & 0.0 \\
$5^{\text {th }}$ week & 128 & 64 & 256 & 128 & 0.0 \\
$6^{\text {th }}$ week & 512 & 256 & 1024 & 512 & 0.0 \\
$7^{\text {th }}$ week & 256 & 128 & 512 & 256 & 0.0 \\
\hline $8^{\text {th }}$ week & 128 & 64 & 128 & 128 & 0.0
\end{tabular}

RA: Riemerella anatipestifer

AI: Avian Influenza

Heamagglutination Inhibition test (HI): It was used for evaluation of humeral immune response against AI. It was performed according to (OIE, 2019).

\section{Challenge test}

The vaccinated and control groups of ducks were challenged with 0.1 of $10^{8} \mathrm{CFU}$ of each $R$. anatipestifer strains RA1 and RA2 intramuscularly (Babars, 2000). Birds after challenge were observed daily for a week for any mortality or clinical signs. The clinical findings of both the vaccinated and unvaccinated birds were observed and recorded.

\section{STATISTICAL ANALYSIS}

P Values of outcome data were calculated and analyzed using SPSS program version 21 (2012).

\section{RESULTS}

RESULTS OF QUALITY CONTROL OF PREPARED VACCINES Purity test: The Culture of $R$. anatipestifer and AI virus used in prepared vaccines were confirmed to be pure.

Sterility test: The prepared vaccines were free from bacterial, fungal and Mycoplasma contamination according to (OIE, 2019).

Safety test: The vaccines were found to be safe and produced no clinical, local, systemic or necropsy findings during two weeks observation period of the injected ducks.

INDIRECT HAEMAGGLUTINATION TEST (IHA) AGAINST $R$. ANATIPESTIFER STRAINS IN VACCINATED DUCKS

As shown in Table (2), the serological test carried out on serum samples obtained from ducks vaccinated with sin 
Table 3: IHA antibody titers in sera of ducklings received booster dose 3 weeks and 8 weeks after primary vaccination of inactivated $R$.anatipestifer and combined $R$. anatipestifer and AI vaccines.

\begin{tabular}{|c|c|c|c|c|c|c|c|c|c|}
\hline \multirow{3}{*}{$\begin{array}{l}\text { Weeks post } \\
\text { boostering }\end{array}$} & \multicolumn{4}{|c|}{$\begin{array}{l}\text { Boostering } 3 \text { weeks post primary vaccina- } \\
\text { tion }\end{array}$} & \multicolumn{4}{|c|}{$\begin{array}{l}\text { Boostering } 8 \text { weeks post primary vac- } \\
\text { cination after the titer decreased }\end{array}$} & \multirow{3}{*}{ Control } \\
\hline & \multicolumn{2}{|c|}{$\begin{array}{l}\text { Inactivated RA } \\
\text { vaccine }\end{array}$} & \multicolumn{2}{|c|}{$\begin{array}{l}\text { Combined inactivated } \\
\text { RA and AI vaccine }\end{array}$} & \multicolumn{2}{|c|}{$\begin{array}{l}\text { Inactivated } \\
\text { RA vaccine }\end{array}$} & \multicolumn{2}{|c|}{$\begin{array}{l}\text { Combined inactivat- } \\
\text { ed RA and AI vaccine }\end{array}$} & \\
\hline & $\mathrm{RA}_{1}$ & $\mathrm{RA}_{2}$ & $\mathrm{RA}_{1}$ & $\mathrm{RA}_{2}$ & $\mathrm{RA}_{1}$ & $\mathrm{RA}_{2}$ & $\mathrm{RA}_{1}$ & $\mathrm{RA}_{2}$ & \\
\hline $1^{\text {st }}$ week PB & 64 & 32 & 128 & 64 & 256 & 128 & 256 & 256 & 0.0 \\
\hline $2^{\text {nd }}$ week PB & 128 & 64 & 256 & 128 & 512 & 256 & 512 & 512 & 0.0 \\
\hline $3^{\text {rd }}$ week PB & 256 & 128 & 512 & 256 & 1024 & 512 & 1024 & 1024 & 0.0 \\
\hline $4^{\text {th }}$ week PB & 512 & 256 & 1024 & 512 & 1024 & 512 & 1024 & 1024 & 0.0 \\
\hline $5^{\text {th }}$ week PB & 512 & 256 & 1024 & 512 & 512 & 256 & 1024 & 1024 & 0.0 \\
\hline $6^{\text {th }}$ week PB & 256 & 128 & 512 & 256 & 256 & 128 & 512 & 512 & 0.0 \\
\hline $7^{\text {th }}$ week PB & 128 & 64 & 256 & 128 & 128 & 64 & 256 & 128 & 0.0 \\
\hline
\end{tabular}

RA: Riemerella anatipestifer AI: Avian Influenza PB: Post boostering

Table 4: $\mathrm{HI} \log _{2}$ titerin sera of ducklings vaccinated with single dose of inactivated AI and combined R. anatipestifer and $\mathrm{AI}$ vaccines.

\begin{tabular}{llll} 
& Type of vaccines & & \\
& Inactivated AI vaccine & Combined inactivated RA and AI vaccine & Control \\
Pre-vaccination & 0 & 0 & 0 \\
$2^{\text {nd }}$ week & 6 & 6.6 & 0 \\
$3^{\text {rd }}$ week & 6.8 & 7.6 & 0 \\
$4^{\text {th }}$ week & 7.4 & 8.2 & 0 \\
$5^{\text {th }}$ week & 8 & 9 & 0 \\
$6^{\text {th }}$ week & 8 & 8.6 & 0 \\
$7^{\text {th }}$ week & 6.2 & 7.8 & 0 \\
$8^{\text {th }}$ week & 5.8 & 6.4 & 0 \\
\hline
\end{tabular}

RA: Riemerella anatipestifer

AI: Avian Influenza

gle dose of inactivated RA and combined RA and AI vaccines revealed that such birds exhibited detectable $R$. anatipestifer antibodies from the second week post vaccination. The antibody titer increased gradually till reached its peak at $6^{\text {th }}$ week post vaccination where it reached 512 and 256 for $\mathrm{RA}_{1}$ and $\mathrm{RA}_{2}$ strains respectively in the $R$. anatipestifer vaccine while 1024 and 512 for $\mathrm{RA}_{1}$ and $\mathrm{RA} \mathrm{A}_{2}$ strains respectively in the combined inactivated $R$. anatipestifer and AI vaccine. The antibodies titer was gradually decreased till reached 128 and 64 for $\mathrm{RA}_{1}$ and $\mathrm{RA}_{2}$ strains respectively in the $R$. anatipestifer vaccine while 128 for both $\mathrm{RA}_{1}$ and $\mathrm{RA}_{2}$ strains in the combined $R$. anatipestifer and $\mathrm{AI}$ vaccine at the $8^{\text {th }}$ week post vaccination. Also from this table, the combined RA and AI vaccine showed higher level of antibody titers when compared with the $R$. anatipestifer vaccine and the antibody titers were decreased at $8^{\text {th }}$ week so this subgroups (c) were received a booster dose of vaccines at $8^{\text {th }}$ week. The $p$ values for R1and R2 strains between mono and combined vaccines were 0.110 and 0.05 respectively.
As shown in Table (3), the inoculation of a booster dose 3 weeks post primary vaccination (subgroup b) caused rapid rise of antibody titers till reached the peak at $4^{\text {th }}$ week post boostering where it reached 512 and 256 for $\mathrm{RA}_{1}$ and $\mathrm{RA}_{2}$ strains respectively in the RA vaccine while 1024 and 512 for $\mathrm{RA}_{1}$ and $\mathrm{RA}$ strains respectively in the combined $\mathrm{RA}$ and AI vaccine that persist for the $5^{\text {th }}$ week then gradually decreased till reached 128,64 for $\mathrm{RA}_{1}$ and $\mathrm{RA}_{2}$ strains respectively in the RA vaccine while 256 and 128 for $R A_{1}$ and $\mathrm{RA}_{2}$ strains respectively in the combined RA and AI vaccine at $7^{\text {th }}$ week post boostering. On the other hand, the inoculation of a booster dose 8 weeks post primary vaccination after the titer was decreased (subgroup c) caused increase in the titer till reached the peak at $3^{\text {rd }}$ week post boostering where it reached 1024 and 512 for $\mathrm{RA}_{1}$ and $\mathrm{RA}_{2}$ strains respectively in the RA vaccine while 1024 in the combined RA and AI vaccine that persist for $4^{\text {th }}$ week in RA vaccine and persist for $5^{\text {th }}$ week in the combined vaccine then gradually decreased till reached 128, 64 for $R A_{1}$ and $R A_{2}$ strains respectively in $R A$ vaccine and 256 
Table 5: $\mathrm{HI} \log _{2}$ titerin sera of ducklings received booster dose 3wks and 8wks after primary vaccination of inactivated $\mathrm{AI}$ and combined $R$. anatipestifer and AI vaccines

\begin{tabular}{|c|c|c|c|c|c|}
\hline \multirow[t]{2}{*}{ Weeks post boostering } & \multicolumn{2}{|c|}{$\begin{array}{l}\text { Boostering } 3 \text { weeks post primary } \\
\text { vaccination }\end{array}$} & \multicolumn{2}{|c|}{$\begin{array}{l}\text { Boostering } 8 \text { weeks post primary vacci- } \\
\text { nation after the titer decreased }\end{array}$} & \multirow[t]{2}{*}{ Control } \\
\hline & $\begin{array}{l}\text { Inactivated } \\
\text { AI vaccine }\end{array}$ & $\begin{array}{l}\text { Combined } R A \text { and } \\
\text { AI vaccine }\end{array}$ & $\begin{array}{l}\text { Inactivated } A I \\
\text { vaccine }\end{array}$ & $\begin{array}{l}\text { Combined RA } \\
\text { and AI vaccine }\end{array}$ & \\
\hline $1^{\text {st }}$ week PB & 7.6 & 8 & 6.8 & 7.4 & 0 \\
\hline $2^{\text {nd }}$ week PB & 8 & 8.4 & 7.7 & 8.2 & 0 \\
\hline $3^{\text {rd }}$ week PB & 8.2 & 8.6 & 8.2 & 8.6 & 0 \\
\hline $4^{\text {th }}$ week PB & 8.4 & 8.7 & 8.4 & 8.8 & 0 \\
\hline $5^{\text {th }}$ week $\mathrm{PB}$ & 8.4 & 9 & 8.6 & 9 & 0 \\
\hline $6^{\text {th }}$ week PB & 8 & 8.4 & 8.4 & 8.7 & 0 \\
\hline $7^{\text {th }}$ week PB & 7.2 & 7.6 & 8 & 8.4 & 0 \\
\hline
\end{tabular}

RA: Riemerella anatipestifer

AI: Avian Influenza

PB: Post boostering

Table 6: Mean protective efficacy in ducks vaccinated with RA and combined RA and AI vaccines that challenged 3 weeks after primary vaccination and 3 weeks post boostering.

\begin{tabular}{|c|c|c|c|c|c|c|c|c|c|c|c|c|}
\hline & \multicolumn{6}{|c|}{$\begin{array}{l}\text { Challenge 3weeks post vaccination (subgroup } \\
\text { a) }\end{array}$} & \multicolumn{6}{|c|}{$\begin{array}{l}\text { Challenge } 3 \text { weeks post boostering } \\
\text { (subgroup b) }\end{array}$} \\
\hline & \multicolumn{2}{|c|}{ RA vaccine } & \multicolumn{2}{|c|}{$\mathrm{RA}+\mathrm{AI}$} & \multicolumn{2}{|c|}{ Control } & \multicolumn{2}{|c|}{ RA vaccine } & \multicolumn{2}{|c|}{$\mathrm{RA}+\mathrm{AI}$} & \multicolumn{2}{|c|}{ Control } \\
\hline & $\mathrm{RA}_{1}$ & $\mathrm{RA}_{2}$ & $\mathrm{R} \mathrm{A}_{1}$ & $\mathrm{RA}_{2}$ & $\mathrm{RA}_{1}$ & $\mathrm{RA}_{2}$ & $\mathrm{RA}_{1}$ & $\mathrm{RA}_{2}$ & $\mathrm{RA}_{1}$ & $\mathrm{RA}_{2}$ & $\mathrm{RA}_{1}$ & $\mathrm{RA}_{2}$ \\
\hline No. of challenged ducks & 20 & 20 & 20 & 20 & 5 & 5 & 20 & 20 & 20 & 20 & 5 & 5 \\
\hline No. of died ducks & 6 & 6 & 5 & 5 & 4 & 4 & 4 & 4 & 3 & 3 & 4 & 4 \\
\hline Protection \% & 70 & 70 & 75 & 75 & 20 & 20 & 80 & 80 & 85 & 85 & 20 & 20 \\
\hline
\end{tabular}

RA: Riemerella anatipestifer AI: Avian Influenza

Subgroup (a): ducks that received one dose of vaccine then challenged after 3 weeks.

Subgroup (b): ducks that received booster dose of vaccine 3 weeks post vaccination then challenged after 3 weeks post boostering.

and 128 for $\mathrm{RA}_{1}$ and $\mathrm{RA}$, strains respectively in combined $\mathrm{RA}$ and $\mathrm{AI}$ vaccines at $7^{\text {th }}$ week post boostering.

Also from this table, the combined RA and AI vaccine showed higher level of antibody titers after inoculation of booster dose when compared with the $R$. anatipestifer vaccine.

The $\mathrm{p}$ values for R1and R2 strains after 3 weeks boostering between mono and combined vaccines were 0.009 for the two strains while they were 0.134 and 0.007 for 8 weeks boostering respectively.

\section{Haemagglutination inhibition test against} AVIAN INFLUENZA IN VACCINATED DUCKS

As shown in Table (4), the serological test carried out on serum samples obtained from ducks vaccinated with single dose of inactivated $\mathrm{AI}$ and combined $\mathrm{RA}$ and $\mathrm{AI}$ vaccines revealed that such birds exhibited detectable $\mathrm{AI}$ antibodies from the second week post vaccination. The antibody titer increased gradually till reached its peak at $5^{\text {th }}$ week post vaccination where it reached $8 \mathrm{HI} \log _{2}$ in $\mathrm{AI}$ vaccine and 9HI $\log _{2}$ in combined RA and AI vaccine that persist for $6^{\text {th }}$ week. The antibodies titer was gradually decreased till reached 5.8 and 6.4 in AI vaccine and combined RA and AI vaccine respectively at $8^{\text {th }}$ week post vaccination. Also from this table, the combined RA and AI vaccine showed higher level of antibody titers when compare d with the $A I$ vaccine and the antibody titers were decreased at $8^{\text {th }}$ week so this subgroup were received a booster dose of vaccines at $8^{\text {th }}$ week. The $p$ values between mono and combined vaccines were 0.001 .

From Table (5), the inoculation of a booster dose 3 weeks post primary vaccination caused rapid rise of antibody titers till reached the peak $8.4 \mathrm{HI} \log _{2}$ in $\mathrm{AI}$ vaccine at $4^{\text {th }}$ week post boostering while it reached its peak $9 \mathrm{HI} \log _{2}$ at $5^{\text {th }}$ week post boostering in combined RA and AI vaccine. The antibody titers persisted for another week then decreased till reached 7.2 in AI vaccine and 7.6 in combined RA and AI vaccine at $7^{\text {th }}$ week post boostering. On the other hand, the inoculation of a booster dose 8 weeks post primary vaccination after the titer was decreased caused increase in the antibody titer till reached the peak $8.6 \mathrm{HI}$ $\log _{2}$ in AI vaccine while it reached its peak $9 \mathrm{HI} \log _{2}$ in combined RA and $\mathrm{AI}$ vaccine at $5^{\text {th }}$ week post boostering. 


\section{OPEN OACCESS}

The antibody titers decreased till reached 8 in AI vaccine and 8.4 in combined RA and AI vaccine at $7^{\text {th }}$ week post boostering.

The $\mathrm{p}$ values between mono and combined vaccines when boostering at 3 weeks and when boostering at 8 weeks were 0.000 .

\section{RESULTS OF CHALLENGE TEST}

As shown in Table (6), evaluation of the protection rate in the vaccinated ducks using challenge test by $R$. anat $i-$ pestifer strains $\left(\mathrm{RA}_{1}\right.$ and $\left.\mathrm{RA}_{2}\right)$ in the (subgroup a) which received single dose then challenged after 3 weeks cleared that in inactivated $\mathrm{RA}$ vaccine, the protection percent was $70 \%$ for both $\mathrm{RA}_{1}$ and $\mathrm{RA}_{2}$ strains. Meanwhile in combined inactivated $\mathrm{RA}$ and $\mathrm{AI}$ vaccine, the protection percent was $75 \%$ for both $\mathrm{RA}_{1}$ and $\mathrm{RA}_{2}$ strains. On the other hand, the protection percent by inoculation of booster dose (subgroup b) was $80 \%$ for both $\mathrm{RA}_{1}$ and $\mathrm{RA}_{2}$ strains in $\mathrm{RA}$ vaccine while it was $85 \%$ for $\mathrm{RA}_{1}$ and $\mathrm{RA}_{2}$ strains in combined RA and AI vaccine 3 weeks post boostering. The protection percent in unvaccinated control group was $20 \%$ in both vaccines.

\section{DISCUSSION}

Riemerella anatipestifer infection (RA) causes major economic losses in duck, through high mortality rates, poor feed conversion, increased condemnations, and high treatment costs (Kardos et al., 2007; Sun et al., 2012).

$R$. anatipestifer causes disease in ducks throughout the world. Formerly known as pasteurella anatipestifer, that usually causes disease in young ducklings aged between 2 and 6 weeks. Stress factors such as moving birds and environmental variations can trigger disease. Signs usually include head shaking, lethargy and an abnormal gait. Post mortem signs in acute cases include enlargement of the liver and spleen and lung congestion. In more chronic cases, pericarditis, perihepatitis and air sacculitis with a caseous deposit can be found (Sandhu, 1986; Higgins et al., 2000).

Control of $R$. anatipestifer and avian influenza is still subject of interest and usually attract attention of researchers to know more about diseases, epidemiology and how to control in susceptible birds. Combination of bacterial and viral vaccines has many benefits for the manufacturer as it reduces production costs, save time, effort and simplifies the immunization schedule. Also it minimizes stress of multiple vaccinations on the animals. Vaccination is still considered one of the major tools for controlling both of the two diseases. Vaccine efficacy depends on many variables, such as the nature, the amount of antigen adminis- trated and the presence of adjuvant to enhance immunogenicity. New resisting adjuvants more and more needed to ensure proper stability profiles of vaccines so, in this study three vaccines were produced, inactivated RA, inactivated $\mathrm{AI}$ and combined inactivated RA and $\mathrm{AI}$ vaccines using a flexible water in oil emulsion Montanide 71VG adjuvant able to resist destabilizing antigenic media without impairing the safety and efficacy of avian vaccine (Arous et al., 2013)

Regarding the potency of the prepared vaccine, the serological tests carried out on serum samples obtained from vaccinated ducks with the combined RA and AI vaccine group (3) revealed that such birds exhibited detectable $R$. anatipestifer antibodies from the second week post vaccination. Evaluation of the antibody titer in sera of the vaccinated ducklings using the IHA test which received single dose, cleared that the superiority of the inactivated combined vaccine than the monovalent vaccine, where the IHA antibody titer increased gradually till reach its maximum level 512, 256, 1024 and 512 in monovalent (RA $)$, $\left(\mathrm{RA}_{2}\right)$ group (1) and combined vaccine $\left(\mathrm{RA}_{1}\right),\left(\mathrm{RA}_{2}\right)$ group (3) respectively at $6^{\text {th }}$ week post first vaccination then gradually decreased till reached 128, 64, 128 and 128 in monovalent $\left(\mathrm{RA}_{1}\right),\left(\mathrm{RA}_{2}\right)$ group (1) and combined vaccine $\left(\mathrm{RA}_{1}\right),\left(\mathrm{RA}_{2}\right)$ group (3), respectively at the $8^{\text {th }}$ week post first vaccination. Also it was noted that combined vaccine showed higher level when compared with the results of ducklings vaccinated with monovalent $R$. anatipestifer. The obtained results agreed with that of, (Amina et al., 2003; AboulSaoud, 2010; Pathansophon et al., 1996) who reported that antibody titers were higher at 5,6 weeks post $1^{\text {st }}$ vaccination.

The inoculation of booster dose 3 weeks apart which caused rapid rise of antibodies titer till reached the peak $512,256,1024$ and 512 in monovalent $\left(\mathrm{RA}_{1}\right),\left(\mathrm{RA}_{2}\right)$ group (1) and combined vaccine $\left(\mathrm{RA}_{1}\right),\left(\mathrm{RA}_{2}\right)$ group (3), respectively at $4^{\text {th }}$ week after booster does that persist for $5^{\text {th }}$ week then gradually decreased till reached $128,64,256$ and 128 in monovalent $\left(\mathrm{RA}_{1}\right),\left(\mathrm{RA}_{2}\right)$ group (1), and combined vaccine $\left(\mathrm{RA}_{1}\right),\left(\mathrm{RA}_{2}\right)$ group (3), respectively at $7^{\text {th }}$ week PB. The obtained results agreed with (Eman et al., 2020).Who noted that all birds showed a secondary response greater than that seen in birds vaccinated once. These findings were in accordance with that recorded by (Jabbri et al., 2005) who stated that a significant immunological stimulus had been elicited by the $2^{\text {nd }}$ exposure and (Iqbal et al., 2010) who reported that the immune response of secondary immunization was significantly higher than the primary vaccination.

Inoculation of $2^{\text {nd }}$ dose after decreasing of antibody titers at the $8^{\text {th }}$ week post primary vaccination stated that antibody titers in the sera of ducklings increased gradually 
till reached the maximum at $3^{\text {th }}$ week $(1024,512,1024$ and 1024) that persist for $4^{\text {th }}$ week in RA vaccine and for $5^{\text {th }}$ week in combined RA and AI vaccine then decreased gradually till reached $128,64,256$ and 128 at $7^{\text {th }}$ week.

Avian influenza (AI) is a major zoonotic viral disease that causes significant adverse impacts on poultry production, the global trade, and public health (Capua et al., 2002; Alexander DJ, 2007). Despite decades of research and control efforts, the incidences and severity of AI outbreaks have not been alleviated but rather increased (CDC, 2017; USDA, 2017).

The most important part of immune response for controlling of AIV is the humeral immunity (Wareing and Tannock, 2001).

The obtained results revealed that $\mathrm{HI}$ titres of birds with single shot of bivalent H5N1 (AI) and Riemerella vaccine group (3) compared to inactivated H5N1 (AI) vaccine group (1) showed gradual increase in the antibody titer where, the maximum $\mathrm{HI}$ titer of birds was $9 \& 8 \log 2 \mathrm{HI}$ titer respectively in $5^{\text {th }}$ week post primary vaccination that persist for $6^{\text {th }}$ week then gradually decreased till reached 6.4 and 5.8 in combined and single vaccines respectively. These results agreed with (Zhao et al., 2017) who reported that the vaccine could induce a fast immunological reaction and the mean $\mathrm{HI}$ titers were over $6.9 \log _{2}$ against both NDV and H9N2 AIV at 2 wpv. At 4 wpv, peak levels of $\mathrm{HI}$ antibodies were detected with a mean HI titer of 8.6 $\log _{2}$ against NDV and $9.5 \log _{2}$ against H9N2.

The results of birds boosted with prepared bivalent H5N1 (AI) and Riemerella vaccine compared to inactivated H5N1 (AI) group (2) vaccine showed the maximum titers $9,8.4 \log _{2}$ respectively at $5^{\text {th }}$ week post boostering 3 weeks after primary vaccination while it was 9 and $8.6 \log _{2}$ post boostering 8 weeks after primary vaccination and decreased gradually till the $7^{\text {th }}$ week. Regarding the statistical analysis, there is a significant difference between inactivated $R A$ vaccine and inactivated combined $R A$ and AI vaccines, also between inactivated $\mathrm{AI}$ vaccine and combined inactivated RA and AI vaccine, these results agree with (Mokhtar et al., 2019) who found that the use of polyvalent vaccines lead to better vaccination coverage.

Regarding the challenge test, the evaluation of the protection rate in the vaccinated ducks using challenge test by $R$. anatipestifer strains $\left(\mathrm{RA}_{1}\right.$ and $\mathrm{RA}_{2}$ ) in the (subgroup a) which received single dose then challenged after 3 weeks cleared that, in inactivated $\mathrm{RA}$ vaccine, the protection percent was $70 \%$ for both $\mathrm{RA}_{1}$ and $\mathrm{RA}_{2}$ strains. Meanwhile in combined inactivated $\mathrm{RA}$ and $\mathrm{AI}$ vaccine, the protection percent was $75 \%$ for both $\mathrm{RA}_{1}$ and $\mathrm{RA}_{2}$ strains. On the other hand, the protection percent by inoculation of
Advances in Animal and Veterinary Sciences booster dose (subgroup b) was $80 \%$ for both $\mathrm{RA}_{1}$ and $\mathrm{RA}_{2}$ strains in $\mathrm{RA}$ vaccine while it was $85 \%$ for $R A_{1}$ and $\mathrm{RA}_{2}$ strains in combined $\mathrm{RA}$ and $\mathrm{AI}$ vaccine 3 weeks post boostering. The protection percent in unvaccinated control group was $20 \%$ in both vaccines.

From these data it was clear that the antibody titer measured with IHA test was highly correlated with protection against challenge with virulent organisms as reported by (Eman et al., 2020). They also stated that the combination increase the antibody titer and protection percent that confirm the obtained results that the protection of ducks vaccinated with combined $R A$ and $A I$ vaccine was higher than $\mathrm{RA}$ vaccine and the protection of ducks that received booster dose was higher than that received single dose.

From mentioned data, we have shown that the use of Montanide ISA 71 VG adjuvant induced high and protective long lasting immunity and this finding was confirmed by (Arous et al., 2013) who obtained high and protective antibodies against Newcastle disease antigen and stated that this new adjuvant has been tested successfully in other vaccines for its ability to resist destabilization conditions, and its safety and efficacy performance have been confirmed and can also be adapted to other veterinary vaccines and species.

In conclusion, single shot of oil based vaccine even it confer protective immune response but boost shot of vaccine conferred the maximum protective immune response for longer duration. Polyvalent vaccine strategies increase reactivity for many pathogens including, but not limited to, influenza although polyvalent vaccine formulations clearly expand the breadth of a single vaccine formulation. The reactivity is still limited to the individual components. The goal of polyvalence is to increase the breadth of vaccine coverage by combining diverse components into a single vaccination. So, the combined vaccine was recommended to used not only to reduce the cost of production of two single vaccines, decrease the stress of two injections, or the two diseases affect the same age and have same signs but also for the significant higher immune response of combined vaccine against both disease to protect both duck breeder and duckling from both pathogens.

\section{ACKNOWLEDGEMENTS}

We appreciate the financial support by Central Laboratory for evaluation of veterinary biologics.

\section{CONFLICT OF INTEREST}

The authors declare that there is no conflict of interest for 
this publication.

\section{AUTHORS CONTRIBUTION}

All authors contributed equally to study design, sampling, methodology, interpretation of results, and writing of the manuscript.

\section{REFERENCES}

-Abdel-Moneim AS, Afifi MA,El-Kady M F (2011). Genetic drift evolution under vaccination pressure among H5N1 Egyptian isolates. Virol. J. 8:283. https:/doi.org/10.1186/1743422X-8-283

-AboulSaoud SA, Manal S Mahmoud, Eman S Ahmed, Amal M EL-Sawah (2010).Immunlogical Studies On preparation and evaluation of Riemerella anatipestifer vaccine in ducks: $10^{\text {th }}$ Sci. Vet. Med. Zag. Conference.

-Alexander DJ (2007). An overview of the epidemiology of avian influenza. Vaccine. 25 (30):5637-44.

-Amina A EL-Bayomy, Eman M EL- Rawy,Manal S Mahmoud, Hassan HA, Daoud A M (2003). Immunological evaluation of avian Pasteurellosis vaccine prepares from local isolates and standard strains. J. Egypt. Vet. Med. Assoc. 63 (4): 2544.

-Arafa AS, Naguib MM, Luttermann C, Selim AA, Kilany WH, Hagag N (2015). Emergence of a novel cluster of influenza $\mathrm{A}(\mathrm{H} 5 \mathrm{~N} 1)$ virus clade 2.2.1.2 with putative human health impact in Egypt. Euro Surveill. 20: 2-8. https:/doi. org/10.2807/1560-7917.ES2015.20.13.21085.

-Arous JB, Deville S, Norkinac S, Borisov V, Rozhdestvenskaia T, Rozhdestvenskiy I, Gaucheron J, Dupuis L (2013). Montanide ${ }^{\mathrm{TM}}$ ISA 71 R VG: A robust and flexible adjuvant formulation for potent and stable poultry vaccines. EggBroiler - India’s Poultry Business Magazine. 6-9.

- Bebars AS (2000). Characterization of pasteurella of duck origin by using protein profile analysis and DNA fingerprinting. M. D. Thesis, Microbiology, Fac. Vet. Med., Cairo Univ.

- Capua I, Mutinelli F, Pozza MD, Donatelli I, Puzelli S, Cancellotti FM (2002). The 1999-2000 avian influenza (H7N1) epidemic in Italy. veterinary and human health implications. Acta Trop. 83(1):7-11.

- Carter GR, Cole JR (1990). Diagnostic procedures in veterinary bacteriology and mycology. $5^{\text {th }}$ Ed. Academic Press, Inc. Harcourt Brance Jovanovich, Publishers, London, New York.

- Cattoli G, Fusaro A,Monne I, Coven F, Joannis T, El-Hamid HS, et al. (2011). Evidence for differing evolutionary dynamics of $\mathrm{A} / \mathrm{H} 5 \mathrm{~N} 1$ viruses among countries applying or not applying avian influenza vaccination in poultry. Vaccine 29: 9368-9375.https:/doi.org/10.1016/j.vaccine.2011.09.127.

- CDC (2017). Avian Influenza Current Situation Summary [updated April 12, 2017; cited 2017 August 28]. https:// www.cdc.gov/flu/avianflu/avian-flu-summary.htm.

- CFR (Code of Federal Regulations) 2019. Title 9: Animals and Animal Products Part 53-Foot-And-Mouth Disease, Pleuropneumonia, and Certain Other Communicable Diseases of livestock Or Poultry.

- Chen YP, Lee SH, Chou CH, Tsai HJ (2012). Detection of florfenicol resistance genes in Riemerella anatipestifer isolates from ducks and geese. Vet. Microbiol. 154: 325-331.
- Chen YP, Tsao MY, Lee SH; Chou CH, Tsai HJ (2010). Prevalence and molecular characterization of chloramphenicol resistance in Riemerella anatipestifer isolates from ducks and geese in Taiwan. Avian Pathol. 39: 333-338.

-Dong-Hun Lee , Chang-Seon Song et al. (2013). H9N2 AIV in Korea: evolution and vaccination. ClinExp Vaccine Res. 2:2633.

-El-Shesheny R, Mostafa A, Kandeil A, Mahmoud SH, Bagato O, Naguib A (2017). Biological characterization of highly pathogenic avian influenza $\mathrm{H} 5 \mathrm{~N} 1$ viruses that infected humans in Egypt in 2014-2015. Arch. Virol. 162: 687-700. https:/doi.org/10.1007/s00705-016-3137-8

-Eman M EL-Rawy, Afaf A Khader, Manal S Mahmoud, Eman S Ahmed, Selim S. Salma, Wafaa S Abd El-Moneim, Abeer Mwafy (2020). Preparation and Evaluation of combined oil adjuvant vaccine against duck pasteurellosis and Riemerella anatipestifer infection in ducks. International Journal of Veterinary Science and Animal Husbandry. 5(1):18-21.

-Higgins DA, Henry RR, Kouner ZV (2000). Duck immune response to Riemrella anatipestifer vaccine. Develop. Comparat. Immunol. 24: 153- 167.

-Hoffmann E, SD Perez, R Webby, RG Webster (2002). Eightplasmid system for rapid generation of influenza virus vaccines. Vaccine. 20:3165-3170.

-Hu Q, Liu X, Miao J, Zhao D, Zhang LndDing C (2001). The epidemiology study of Riemerella anatipestifer infection in Jiangsu and Anhui provinces. Guide Chin. Poult.18: 18-19.

-Iqbal MT, MH Haque, S Sarker, MA Islam, KA Choudhury (2010). Determination of immune response against alumprecipitated fowl cholera vaccine in the quail, coturnix japonica. Univ. J. Zool. Rajshahi. 29:57-60.

-Jabbri AR, GR MoazeniJula (2005). Fowl cholera: Evaluation of a trivalent pasturella multocida vaccine consisted of serotypes 1, 3 and 4. Arch. Razi Ins. 59:103-11.

-Kardos G, Nagy J, Antal M, Bistyak A,Tenk M, Kiss I (2007). Development of a noval PCR assay specific for Riemerella anatipestifer. Lett. Appl. Microbiol. 44: 145-148.

- Liu M, JM Wood, T Ellis, S Krauss, P Seiler, C Johnson, E Hoffmann, J Humberd, D Hulsem, Y Zhang, RG Webster, DR Perez (2003). Preparation of a standardized, efficacious agricultural $\mathrm{H} 5 \mathrm{~N} 3$ vaccine by reverse genetics. Virology. 314:580-590.

- Middleton D et al. (2007). Efficacy of inactivated vaccines against $\mathrm{H} 5 \mathrm{~N} 1$ avian influenza infection in ducks. Virology. 359 (1): 66-71.

- Mokhtar Rizk Gomaa, Ahmed Ali Khalil, Ahmed Kandeil, Jamal SM Sabir,' Ahmed Kayed, Yassmin Moatasim, Marwa F El saied,'Mounir M El-safty, 'Ghazi Kayali,'Mohamed A Ali (2019). Development of an effective contemporary trivalent avian influenza vaccine against circulating $\mathrm{H} 5 \mathrm{~N} 1$, $\mathrm{H} 5 \mathrm{~N} 8$, and H9N2 in Egypt. Poultry Science. 98 (12): 6289-6295 https://doi.org/10.3382/ps/pez385.

- NaguibMM, Abdelwhab EM,HarderTC (2016a).Evolutionary features of influenza $\mathrm{A} / \mathrm{H} 5 \mathrm{~N} 1$ virus populations in Egypt: poultry and human health implications. Arch. Virol. 161: 1963-1967. https:/doi.org/10.1007/s00705-016-2849-0

- Naguib M M, Hagag N, El-Sanousi AA, Hussein H A, Arafa AS (2016b). The matrix gene of influenza A H5N1 in Egypt, 2006-2016: molecular insights and distribution of amantadine-resistant variants. Virus Genes. 52: 872-876. https:/doi.org/10.1007/s11262-016-1373-3

- OIE (2019). Manual of Diagnostic Tests and Vaccines for 
Terrestrial Animals chapter 3.3.4 Avian Influenza Virus.

-Pathanasophon P, Sawada T, Pramoolsinsap T, Tanticharoenyos $T$ (1996). Immunogenicity of Riemerella anatipestifer broth culture bacterin and cell- free culture filtrate in ducks. Avian Pathol. 25: 705-719.

- Rohaim MA, El-Naggar RF, Hamoud MM, Nasr SA, Ismael E, Laban SE (2017). Re-emergence of a novel H5N1 Avian influenza virus variant subclade 2.2.1.1 in Egypt during 2014. Transbound. Emerg. Dis. 64: 1306-1312. https:/doi. org/10.1111/tbed.12472.

- Salaheldin AH, Veits J, Abd El-Hamid HS, Harder TC, Devrishov D, Mettenleiter TC, et al. (2017). Isolation and genetic characterization of a novel 2.2.1.2a H5N1 virus from a vaccinated meat-turkeys flock in Egypt. Virol. J. 14:48. https:/doi.org/10.1186/s12985-017-0697-

- Sandhu TS (1986): Important diseases of d Sandhu TS (1986). Important diseases of duck, p 111-134. In Farrell DJ, Stapleton P (ed), Duck production science and world practice. University of New England, Armidale, Australia.

- Sandhu T (1979). Immunization of white Pekin duckling against Pasteurella anatipesifer infection. Avian Dis. 23: 662-669.

-Smith GJ, Donis RO (2015). World Health Organization/ World Organisation for Animal Health/Food and Agriculture Organization, and (WHO/OIE/FAO) H5 Evolution Working GroupNomenclature updates resulting from the evolution of avian influenza $\mathrm{A}(\mathrm{H} 5)$ virus clades 2.1.3.2a, 2.2.1, and 2.3.4 during 2013-2014. Influenza Other Respir. Viruses 9: 271-276. https:/doi.org/10.1111/ irv. 12324

-Suarez DL, Lee CW, Swayne DE (2006). Avian influenza vaccination in North America: strategies and difficulties.

DevBiol (Basel).124:117-24.

-Subbarao K, Katz JM (2004). Influenza vaccines generated by reverse genetics. Curr Top Microbiol Immunol. 283:313-42.

-Subramaniam S, Huang B, Loh H, Tan H-M, Chua K-L, Frey J, Kwang J (2000). Characterization of a predominant immunogenic outer membrane protein of Riemerella anatipestifer. Clin.Diagn. Lab. Immunol. 7: 168-174.

-Sun N, Liu JH, Yang F, Lin DC, Li GH, Chen ZL, Zeng ZL (2012). Molecular characterization of the antimicrobial resistance of Riemerella anatipestifer isolated from ducks. Vet. Microbiol.158: 376-383.

-USDA (2017). Avian influenza [cited 2017 August 28]. https:// www.usda.gov/topics/animals/one-health/avian-influenza.

-Wareing MD, Tannock GA (2001) . Live attenuated vaccines against influenza; an historical review. Vaccine.19: 3320 3330

-WHO (2017). Cumulative Number of Confirmed Human Cases of Avian Influenza A (H5N1) Reported to WHO. Available online at: http://www.who.int/influenza/human_ animal_interface/H5N1_cumulative_table_archives/en/ (Accessed December 10, 2017).

-Younan M, Poh MK, Elassal E, Davis T, Rivailler P, Balish, AL et al. (2013). Microevolution of highly pathogenic avian influenza $\mathrm{A}(\mathrm{H} 5 \mathrm{~N} 1)$ viruses isolated from humans, Egypt, 2007-2011. Emerg. Infect. Dis. 19: 43-50. https:/doi. org/10.3201/eid1901.121080

-Zhao J, Yang H, Xu H, Ma Z, Zhang G (2017). Efficacy of an inactivated bivalent vaccine against the prevalent strains of Newcastle disease and H9N2 avian influenza.Virology Journal.14:56. https:/doi.org/10.1186/s12985-017-0723-7 